23

\title{
Преобразование данных лазерной ультразвуковой томографии в медицинский формат DICOM
}

\author{
(C) М.М. Новиков ${ }^{1}$, И.В. Решетов ${ }^{2}$, В.А. Симонова ${ }^{1}$, А.С. Бычков ${ }^{3}$, \\ А.А. Карабутов ${ }^{1,3}$, С.А. Черебыло ${ }^{1}$, Д.С. Святославов ${ }^{2, \uparrow}$ \\ ${ }^{1}$ Институт проблем лазерных и информационных технологий РАН - фрилиал Федерального государственного \\ учреждения „Федеральный научно-исследовательский центр „Кристаллография и фотоника“ РАН, \\ 140700 Шатура, Россия \\ ${ }^{2}$ ФГАОУ ВО Первый МГМУ им. И.М. Сеченова Минздрава России (Сеченовский Университет), \\ 119991 Москва, Россия \\ ${ }^{3}$ НИТУ МИСиС - Национальный исследовательский технологический университет МИСиС, \\ 119049 Москва, Россия \\ e-mail: dssvyatoslavov@gmail.com
}

Поступила в редакцию 10.12.2019 г.

В окончательной редакции 11.03.2020 г.

Принята к публикации 28.03.2020 г.

Разработана экспериментальная установка для лазерно-акустической томографии биологических тканей. Проведены экспериментальные исследования фантомных образцов. Разработана программа для преобразования графических данных лазерно-акустической томографии в стандартный медицинских формат.

Ключевые слова: лазерная ультразвуковая томография, формат DICOM, биологическая ткань, цифровая модель, графические данные.

DOI: $10.21883 / O S .2020 .07 .49580 .121-20$

\section{Введение}

Современная цифровая медицина, позволяющая повышать доступность, качество и эффективность медицинской помощи, - один из приоритетных проектов стратегического развития российского здравоохранения, разработанных Минздравом РФ. Внедрение в практику здравоохранения информационных технологий значительно изменяет способы диагностики и лечения, формы взаимодействия врачей с пациентами и коллегами, организацию лечения и восстановления здоровья.

Развитие современного трехмерного моделирования и внедрение нового поколения спиральных компьютерных томографов значительно расширило возможности использования этих информационных технологий в реконструктивной хирургии [1-4]. Специализированное программное обеспечение позволяет оперативно производить обработку томографических данных пациента и создавать цифровые трехмерные модели любого дефекта или зоны патологии. Цифровые модели все активнее используются при подготовке и планировании оперативного вмешательства в челюстно-лицевой хирургии, хирургии шеи и позвоночника, торакальной хирургии, ортопедии и нейрохирургии. Их можно напрямую использовать для создания копий патологий и дефектов конкретного пациента с помощью аддитивных технологий $[1,5]$. Персонифицированные пластиковые копии анатомических структур позволяют проводить эффективное предварительное планирование сложных операций. На основе цифровых моделей дефектов или зон патологий конкретных заболеваний человека можно создавать единые базы данных для интеллектуальных систем поддержки принятия врачебных решений.

\section{Проблемы формирования цифровых моделей по графической информации}

Основное назначение томографического исследования - это медицинская диагностика, уточнение диагноза или отслеживание результатов лечения. Она позволяет выявить наличие патологии и получить о ней дополнительную информацию. В большинстве случаев это делается путем последовательного визуального анализа изображения срезов, сформированных компьютерным томографом. В настоящее время уже доступно формирование трехмерной реконструкции по томографическим данным [6], которая позволяет значительно улучшить выявление патологий и проводить предварительное планирование сложных хирургических операций. Однако создание трехмерной реконструкции требует решения целого ряда задач, поскольку интересующие структуры и окружающие их ткани имеют сходную плотность и плохо различаются на томографических изображениях [6-8]. В подобных случаях возникает необходимость в сегментации - программном выделении интересующих структур в требуемых границах с их последующей визуализацией, анализом и экспортом в виде файла цифровой модели.

Цифровая модель открывает такие дополнительные возможности как: 
- предоперационное моделирование высокоточных шаблонов и имплантатов на основе томографических данных конкретного пациента;

- изготовление с помощью аддитивных технологий копий дефектов и патологий для дальнейшего анализа и планирования операции;

- трехмерная (3D) печать биологических тканей и матриксов для непосредственного восстановления дефекта.

Для решения задачи повышения точности цифровых моделей и развития автоматических методов выделения биологических тканей используется совместное использование различных методов диагностики [7,8]. В настоящее время проводятся исследования по использованию различных методов ультразвуковой диагностики, которая является более безопасной альтернативой рентгеновским лучам для визуализации и сегментации биологических тканей [9-12].

В настоящей работе описывается возможность применения лазерной ультразвуковой томографии для получения цифровых моделей тестовых объектов.

\section{Лазерная ультразвуковая томография}

В традиционном ультразвуковом эхо-импульсном методе короткий акустический зондирующий импульс используется для озвучивания объекта, а зарегистрированное обратно рассеянное акустическое поле - для восстановления внешней границы и внутренних акустических неоднородностей (рассеивателей) [13]. Лазерный ультразвуковой (ЛУ) метод диагностики основан на генерации звуковых волн лазерным импульсом. Этот импульс поглощается в поверхностном слое исследуемого объекта или в специальном материале, называемым оптико-акустическим (ОА) генератором. Временная форма и амплитуда зондирующего ультразвукового импульса, возбужденного ОА генератором, определяются коэффициентом поглощения света в генераторе, временной зависимостью интенсивности лазерного импульса и плотностью поглощенной лазерной энергии.

ЛУ метод похож на традиционный ультразвуковой метод контроля, однако вместо использования пьезоэлектрических преобразователей для генерации и приема короткого акустического зондирующего импульса в ЛУ методе используется короткий (обычно < $10 \mathrm{~ns}$ ) лазерный импульс для генерации короткого ( $<100 \mathrm{~ns})$ и широкополосного (1.9 MHz) акустического зондирующего импульса за счет оптико-акустического эффекта [13]. В этом случае хорошо задемпфированные пьезоэлектрические преобразователи могут использоваться для приема обратно рассеянного акустического поля в широком диапазоне частот [13-15]. ЛУ метод - эффективный и быстродействующий, поскольку для генерации зондирующего ультразвукового сигнала используется лазерный импульс, а ширина полосы зондирующего импульса может быть достаточно широкой (от единиц до десятков $\mathrm{MHz}$ ). При этом ультразвуковые преобразователи могут быть оптимизированы для широкополосного приема и лучшей акустической связи, поскольку они не используются для генерации ультразвука. Это позволяет проводить широкополосный ультразвуковой контроль объектов и эффективно разделять генерацию и обнаружение ультразвуковых сигналов для увеличения отношения сигнал/шум. Кроме того, поперечный профиль акустического пучка повторяет гладкий поперечный профиль освещающего лазерного луча, таким образом практически устраняя боковые лепестки [14].

Появление фокусированных пьезоэлементных антенных решеток и активное развитие средств вычислительной техники существенно расширяют возможности ЛУ томографии внутренней структуры горных пород, композитных материалов, биологических и других гетерогенных сред [16-19]. Высокая сложность построения ЛУ томографических систем требует разработки критериев и методик их сравнения на этапе проектирования и калибровки после изготовления. Основными характеристиками томографических систем являются размеры области чувствительности (области, в которой построенное изображение позволяет надежно определить наличие источника сигнала), пространственное разрешение (минимальное расстояние, на котором два точечных источника различимы на изображении) и глубина резкости (размер области, где достигается высокое пространственное разрешение). Увеличение размера области чувствительности и глубины резкости, а также улучшение пространственного разрешения являются одной из основных целей при проектировании ЛУ томографических систем под конкретную задачу.

На текущий момент экспериментальные системы на основе оптико-акустической томографии только разрабатываются для различных биомедицинских задач (диагностика раковых заболеваний человека, исследование отдельных органов и томографии мелких животных) $[18,19]$. Участники проекта уже разработали методы применения ЛУ томографии для иммерсионной ультразвуковой профилометрии твердых объектов [20,21], измерений внутренней геометрии твердых образцов со сложной геометрией наружной поверхности [22], контроля толстых сварных соединений [23] и оценки внутренней структуры образцов горных пород [24]. ЛУ томография была применена для иммерсионных ультразвуковых исследований биологических объектов в работах [25-27] (часто в сочетании с ОА томографией).

\section{Экспериментальная установка}

В большинстве задач диагностики биологических объектов и неразрушающего контроля актуальна 2Dвизуализация неоднородностей в режиме реального времени. Построение и анализ 3D-изображений в режиме реального времени сложен, требует значительных вы- 


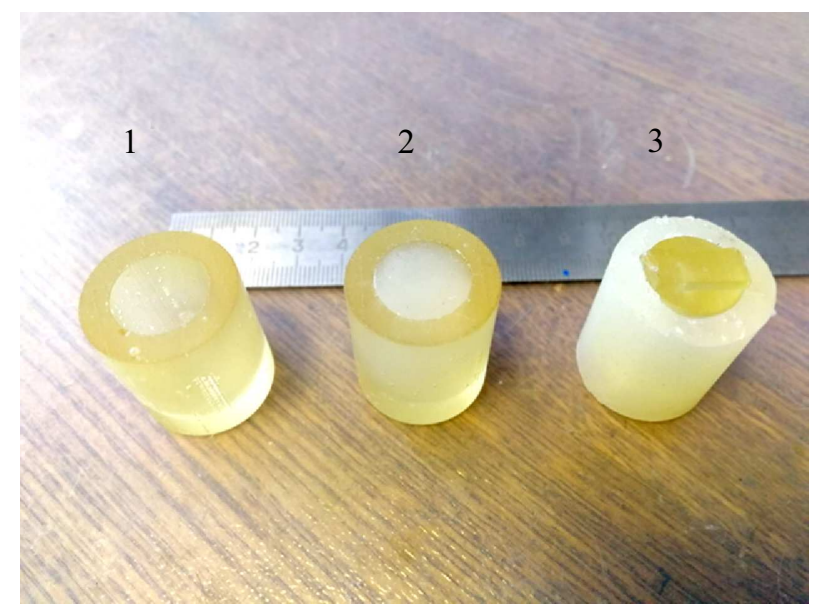

Рис. 1. Фотография тестовых образцов, изготовленных методом лазерной стереолитографии из фотополимера: 1 пустотелый цилиндр, 2 - цилиндр, наполненный силиконом, 3 - силиконовая внешняя оболочка.

числительных мощностей и является самостоятельной задачей.

Для задачи конвертирования данных в стандартный медицинский формат DICOM были созданы и исследованы с помощью ЛУ томографии специальные тестовые образцы с заданной геометрической структурой (рис. 1). Высота цилиндров $20 \mathrm{~mm}$, толщина цилиндрической оболочки для всех образцов $4 \mathrm{~mm}$.

Геометрические образцы заданной формы использовались для настройки и предварительной калибровки ЛУ. Силиконовые вставки применялись для имитации разных сред при распространении ультразвукового сигнала.

Для сканирования биологических тканей была разработана многоканальная комбинированная ЛУ и ОА система. Созданная экспериментальная многоканальная система имеет два режима работы: ЛУ и ОА. Однако для поставленной задачи установка используется только в ЛУ режиме работы. В этом режиме лазерное излучение поглощается в веществе ОА генератора. За счет поглощения лазерного излучения происходит нестационарное расширение материала ОА генератора, что приводит к генерации широкополосных ультразвуковых импульсов. Ультразвуковые импульсы фокусируются акустической линзой на поверхности объекта. Рассеянный на неоднородностях иммерсионной жидкости и на поверхности исследуемого объекта ультразвуковой пучок фокусируется той же акустической линзой на поверхность пьезоэлектрической приемной антенны и регистрируется 16 приемными элементами. Пьезоэлектрические приемные элементы антенны изготовлены из полимерного материала ПВДФ и имеют спектральную полосу приема 1.6-9 MНz. Такая ширина полосы приема обеспечивает разрешение по глубине $\Delta x=0.1 \mathrm{~mm}$. Фокальное расстояние многоэлементной антенны составляло $R=40 \mathrm{~mm}$. Схема экспериментальной установки изображена на рис. 2.
Экспериментальная установка содержит Nd:YAGлазер с модуляцией добротности (Quantel, USA), $\lambda=1064 \mathrm{~nm}$, частотой следования импульсов $20 \mathrm{~Hz}$ и энергией импульса $10 \mathrm{~mJ}$; систему доставки лазерного излучения, включающую оптическое волокно; комбинированный блок питания, обеспечивающий электропитание приемной антенны и усилителя принимаемых электрических сигналов; скоростную многоканальную систему сбора и обработки экспериментальных данных на основе архитектуры NI FlexRIO, содержащую прецизионный аналого-цифровой преобразователь (частота дискретизации $50 \mathrm{Mhz}$ ) и обеспечивающую перевод электрических сигналов с пьезоэлектрических приемных элементов в цифровую форму, их запоминание, усреднение и передачу по высокоскоростной линии связи в персональный компьютер; систему обработки данных, включающую персональный компьютер, связанный скоростной линией передачи данных с системой сбора и обработки экспериментальных данных, программноаппаратную платформу CUDA (Compute Unified Device Architecture) и соответствующее программное обеспечение, осуществляющую прием цифровых данных, их обработку и построение изображения исследуемого объекта, а также интерактивное управление процессом передачи и обработки данных; приемную антенну, содержащую оптико-акустический преобразователь (ОА-генератор) и решетку широкополосных пьезоэлектрических приемных элементов; систему автоматизированного трехмерного позиционирования приемной антенны и исследуемого объекта. Система позволяет изменять относительное положение объекта в держателе с точностью $0.01 \mathrm{~mm}$.

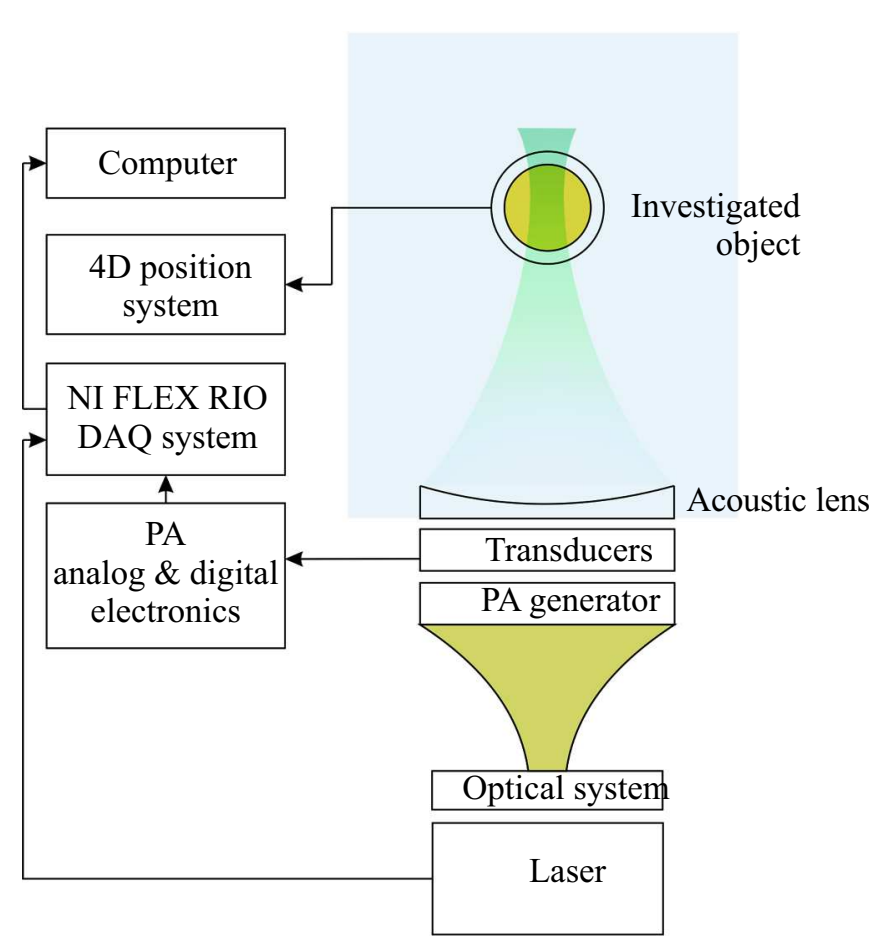

Рис. 2. Схема экспериментальной установки. 

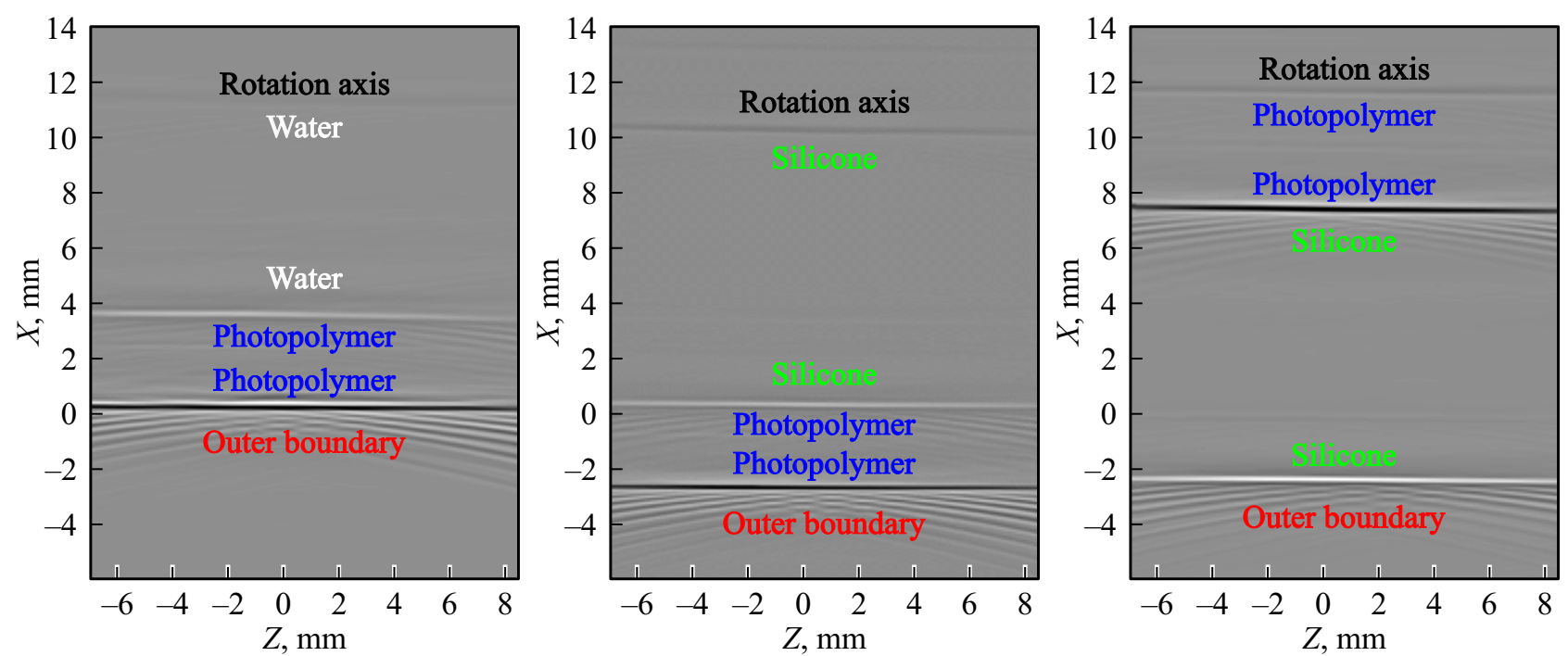

Рис. 3. 2D изображение тестовых образов № 1, № 2 и № 3 (слева направо) с отмеченными границами слоев.

Для управления экспериментальной установкой было разработано специализированное программное обеспечение с графическим интерфейсом, позволяющим управлять движением образца. Интерфейс отображает в режиме реального времени (частота обновления $10 \mathrm{~Hz}$ ) томограммы рассеивателей, находящихся в фокальной плоскости антенны. Для построения томограмм используется алгоритм фильтрованных обратных проекций.

\section{Результаты исследования тестовых образцов}

В эксперименте тестовые объекты погружались в иммерсионную жидкость (вода) и поворачивались вокруг своей оси (ось цилиндра). 2D-изображения каждого тестового объекта снимались с угловым шагом $10^{\circ}$ (рис. 3). Далее изображение фильтровалось и сшивалось в псевдо-3D-изображение для каждого тестового объекта (2D-изображение в декартовой системе координат).

На полученных изображениях (рис. 3) хорошо видны границы аддитивно спеченного фотополимера, а также силиконовой вставки. Видно, что изображение нуждается в дополнительной фильтрации, так как хорошо заметны „артефакты“ - дуги около внешней поверхности объекта. Однако даже на данный момент можно четко определить, что ЛУ метод хорошо применим для контроля как поверхности аддитивно спеченного пластика, так и для контроля его внутренней структуры, так как на изображении хорошо видны неоднородности аддитивно спеченного слоя пластика (непроклеи, неровности слоев). На рис. 3 хорошо видно различие в толщине силикона и пластика на ЛУ изображениях (более, чем в 2 раза). Это связано с отсутствием учета различия скоростей распространения звука в различных материалах при восстановлении изображений. В связи с этим нужно вносить дополнительные коррективы в алгоритм восстановления изображения. Необходимо учитывать различную скорость распространения ультразвука в различных слоях, для корректной оценки расстояний между границами, соответствующими реальным толщинам образца.

\section{Преобразование графических данных в формат DICOM}

Собранные с экспериментальной установки данные были сохранены в формате MATLAB.MAT. Для последующего использования в программах обработки на языке C++ была использована библиотека Matio (an open-source $\mathrm{C}$ library for reading and writing binary MATLAB MAT files under a Simplified BSD license. https://sourceforge.net/p/matio). Результаты, полученные на установке, представляют собой 2D-данные, представленные в цилиндрической системе координат. Конвертация в декартову систему координат производилась посредством обхода пикселей целевого изображения в декартовой системе координат с вычислением положения исходной точки и ближайших к ней пикселей в цилиндрической системе координат с последующей интерполяцией (рис. 4).

При переводе полученных изображений в декартовой системе координат в формат DICOM были использованы библиотеки в исходных кодах DICOM ToolKit (DCMTK, https:/dcmtk.org/dcmtk.php.en) и класс CxImage (https://www.codeproject.com/Articles/1300/CxImage) под собственными свободными лицензиями.

DICOM - медицинский отраслевой стандарт создания, хранения, передачи и визуализации цифровых медицинских изображений и документов обследованных пациентов [28]. DICOM-файл - объектно-ориентированный файл с теговой организацией, информационная 

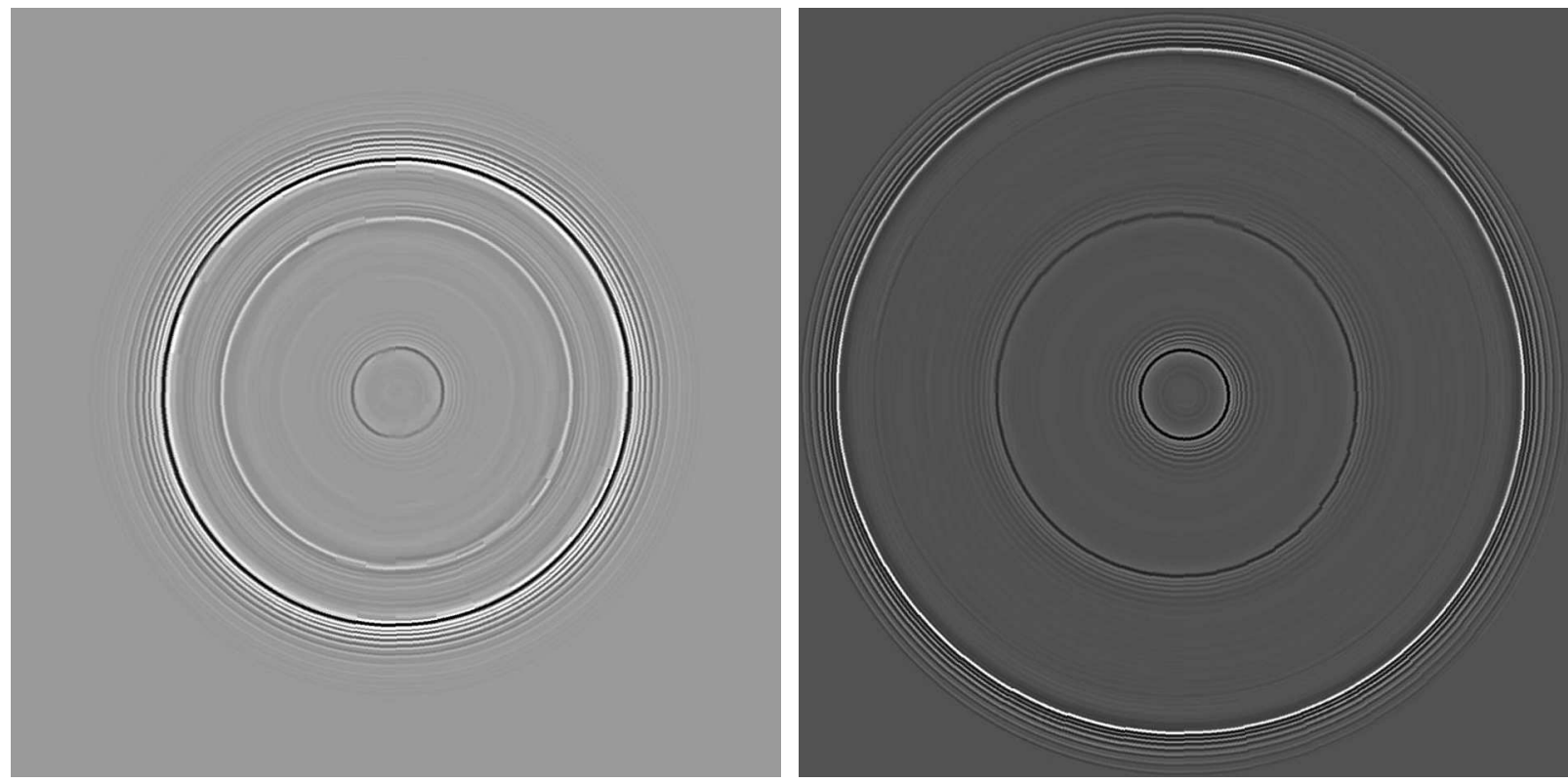

Рис. 4. Конвертированные в декартову систему координат 2D изображения тестовых образцов № 1 (слева) и № 3 (справа) (формат изображения $720 \times 720$ пикселей).

модель стандарта DICOM четырёхступенчатая: пациент $($ patient $) \rightarrow$ исследование $($ study) $\rightarrow$ серия $($ series $) \rightarrow$ изображение (кадр или серия кадров, image). Стандарт DICOM описывает „паспортные“ данные пациента, условия проведения исследования, положение пациента в момент получения изображения ит.п., для того чтобы в последствии было возможно провести медицинскую интерпретацию данного изображения.

Проблема использования дополнительной фильтрации изображения для выделения границ сред и удаления артефактов остается предметом последующего изучения. Также планируются проведения исследований и калибровки геометрической размерности, получаемой графической информации, что очень важно для построения точных цифровых моделей для медицины.

\section{Заключение}

Учитывая обзор работ в области ЛУ томографии, в рамках проекта проведена модификация и оптимизация экспериментальной установки для исследования биологических объектов. Подготовлены и исследованы тестовые образцы. Разработано программное обеспечение для обработки 2D-изображений и их конвертации в декартову систему координат. Конвертация производится посредством обхода пикселей целевого изображения в декартовой системе координат с вычислением положения исходной точки и ближайших к ней пикселей в цилиндрической системе координат с последующей интерполяцией. Разработан специальный транслятор графических данных ЛУ томографии в стандарт DICOM. Следующей задачей данной работы является проведение исследования методом ЛУ томографии биологических объектов и получение цифровых моделей хрящевых структур. Разработка специализированных инструментов сегментации и улучшения их функционала с совместным использованием результатов различных видов медицинской диагностики (компьютерная томография, магнитнорезонансная томография) и ЛУ томографии.

\section{Финансирование работы}

Работа подержана Российским фондом фундаментальных исследований проект № 18-29-03238. Работа по лазерной стереолитографии выполнена при финансовой поддержке Министерства науки и высшего образования (Государственное задание ФНИЦ „Кристаллография и фотоника“ РАН).

\section{Конфликт интересов}

Авторы заявляют об отсутствии конфликта интересов. Авторы несут ответственность за оригинальность представленных данных и возможность публикации иллюстративного материала: таблиц, рисунков, фотографий.

\section{Список литературы}

[1] Ипполитов Е.В., Новиков М.М., Черебыло С.А., Евсеeв A.B. // Современные лазерно-информационные технологии / Под ред. В.Я. Панченко, Ф.В. Лебедева. М.: Интерконтакт Наука, 2015. С. 358-373, ISBN 978-5902063-52-0

[2] Guang-Ye Wang, Wen-Jun Huang, Qi Song, Yun-Tian Qin, Jin-Feng Liang // Computer Assisted Surgery. 2016. V. 21 N 1. P. 160-165 doi 10.1080/24699322.2016.1240235 
[3] Mirzoev M.Sh., Shakirov M.N., Khushvakhtov D.I., Dzhonibekova R.N., Grigoryev E.G. // Avicenna Bulletin. 2018. V. 20. N 2-3. P. 170-175.

[4] van Eijnatten M., Koivisto J., Karhu K., Forouzanfar T., Wolff J. // J. CARS. 2017. V. 12. P. 607-615. doi 10.1007/s11548-016-1490-4

[5] Javaid M., Haleem A. // Alexandria J. Medicine. 2018. V. 54. P. 411-422. doi 10.1016/j.ajme.2017.09.003

[6] Новиков М.М., Внук В.В., Ипполитов Е.В., Черебыло С.A. // Материалы Международной конференции $\mathrm{NT}+\mathrm{M} \&$ Ec. 2019. C. 26-31.

[7] Соловьева С.Н., Уросова В.С. // Современные наукоемкие технологии. 2018. № 5. С. 145-157.

[8] Musatian S.A., Lomakin A.V., Sartasov S.Yu., Popyvanov L.K., Monakhov I.B., Chizhova A.S. // Trudy ISP RAN/Proc. ISP RAS. 2018. V. 30. N 4. P. 183-194. doi 10.15514/ISPRAS-2018-30(4)-12

[9] Machado I., Toews M., Luo J., Unadkat P., Essayed W., George E., Teodoro P., Carvalho H., Martins J., Golland P., Pieper S., Frisken S., Golby A., Wells W. // International J. Computer Assisted Radiology and Surgery. 2018. V. 13. P. 1525-1538. doi 10.1007/s11548-018-1786-7

[10] Mingjian Sun, Depeng Hu, Wenxue Zhou, Yang Liu, Yawei Qu, Liyong Ma // Appl. Sci. 2019. V. 9. P. 1904. doi 10.3390/app9091904

[11] Villa M., Dardenne G., Nasan M., Letissier H., Hamitouche C., EStindel E. // International J. Computer Assisted Radiology and Surgery. 2018. V. 13. P. 1707-1716. doi 10.1007/s11548-018-1856-x

[12] Pandey P., Guy P., Hodgson A.J., Abugharbieh R. // International J. Computer Assisted Radiology and Surgery. 2018. V. 13. P. $1515-1524$. doi 10.1007/s11548-018-1788-5

[13] Oraevsky A., Karabutov A. // Proc. SPIE. 2000. V. 3916. P. 228-239. doi 10.1117/12.386326

[14] Bychkov A., Simonova V., Zarubin V., Cherepetskaya E., Karabutov A. // Appl. Sci. 2018. V. $8 . \quad$ P. 1931. doi 10.3390/app8101931

[15] Karabutov A.A., Podymova N.B., Letokhov V.S. // Appl. Phys. B. 1996. V. 63. P. 545-563. doi 10.1007/BF01830994

[16] Khokhlova T.D., Pelivanov I.M., Karabutov A.A. // Appl. Phys. Lett. 2008. V. 92. 024105. doi 10.1063/1.2834855

[17] Bychkov A.S., Cherepetskaya E.B., Karabutov A.A., Makarov V.A. // J. Biomed. Opt. 2017. V. 22. 076003. doi 10.1117/1.JBO.22.7.076003

[18] Schellenberg M.W., Hunt H.K. // Photoacoustics. 2018. V. 11. P. 14-27. doi 10.1016/j.pacs.2018.07.001

[19] Beard P. // Interface Focus. 2011. V. 1. P. 602-631. doi 10.1098/rsfs.2011.0028

[20] Zarubin V., Bychkov A., Karabutov A., et al. // Appl. Opt. 2018 V. 57. C. $70-76$.

[21] Zarubin V., Bychkov A., Karabutov A., Simonova V., Cherepetskaya E. // In MATEC Web Conf. 2018. V. 145. P. 05009. doi 10.1051/matecconf $/ 201814505009$

[22] Zarubin V., Bychkov A., Simonova V., Zhigarkov V., Karabutov A., Cherepetskaya E. // Appl. Phys. Lett. 2018. V. 112. P. 214102. doi 10.1063/1.5030586

[23] Shibaev I.A., Cherepetskaya E.B., Bychkov A.S., Zarubin V.P. // IJCIET. 2018. V. 9. P. 84-92.

[24] Bychkov A.S., Zarubin V.P., Karabutov A.A., Simonova V.A. // Photoacoustics. 2017. V. 5. P. 10-16. doi 10.1016/j.pacs.2017.01.002
[25] Jo J., Tian C., Xu G., Sarazin J., Schiopu E., Gandikota G., Wang $X$. // Photoacoustics. 2018. V. 12. P. 82-89. doi 10.1016/j.pacs.2018.07.004

[26] Buchmann J., Kaplan B., Powell S., Prohaska S., Laufer J. // Photoacoustics. 2020. V. 17. P. 100157. doi 10.1016/j.pacs.2019.100157

[27] Johnson J.L., Shragge J., van Wijk K. // J. Biomed. Opt. 2017. V. 22. N 4. 041014. doi 10.1117/1.JBO.22.4.041014

[28] Digital Imaging and Communications in Medicine (DICOM), National Electrical Manufacturers Association, Rosslyn, USA, Режим доступа: https://www.dicomstandard.org/current/ 